\title{
KCa3.1 Mediates Dysregulation of Mitochondrial Quality Control in Diabetic Kidney Disease
}

\author{
Chunling Huang ${ }^{1}$, Hao Yi'1, Ying Shi' ${ }^{2}$, Qinghua Cao', Yin Shi', Delfine Cheng ${ }^{3}$, \\ Filip Braet ${ }^{3,4}$, Xin-Ming Chen ${ }^{1}$ and Carol A. Pollock ${ }^{1 *}$
}

${ }^{1}$ Kolling Institute, Sydney Medical School Northern, Faculty of Medicine and Health, University of Sydney, Royal North Shore Hospital, Sydney, NSW, Australia, ${ }^{2}$ Division of Nephrology, School of Medicine, Stanford University, Stanford, CA, United States, ${ }^{3}$ Discipline of Anatomy and Histology, School of Medical Sciences, Faculty of Medicine and Health, The Bosch Institute, University of Sydney, Sydney, NSW, Australia, ${ }^{4}$ Australian Centre for Microscopy and Microanalysis, University of Sydney, Sydney, NSW, Australia

\section{OPEN ACCESS}

Edited by:

Noah Lucas Weisleder

The Ohio State University,

United States

Reviewed by:

Hao Zhou,

People's Liberation Army General Hospital, China

Chengyuan Tang,

Central South University, China

${ }^{*}$ Correspondence: Carol A. Pollock

carol.pollock@sydney.edu.au

Specialty section:

This article was submitted to

Molecular Medicine,

a section of the journal

Frontiers in Cell and Developmental

Biology

Received: 18 June 2020 Accepted: 03 February 2021

Published: 19 February 2021

Citation:

Huang $C$, Yi H, Shi Y, Cao Q, Shi Y, Cheng D, Braet F, Chen X-M and Pollock CA (2021) KCa3.1

Mediates Dysregulation of Mitochondrial Quality Control

in Diabetic Kidney Disease. Front. Cell Dev. Biol. 9:573814. doi: 10.3389/fcell.2021.573814
Mitochondrial dysfunction is implicated in the pathogenesis of diabetic kidney disease. Mitochondrial quality control is primarily mediated by mitochondrial turnover and repair through mitochondrial fission/fusion and mitophagy. We have previously shown that blockade of the calcium-activated potassium channel KCa3.1 ameliorates diabetic renal fibrosis. However, the mechanistic link between $\mathrm{KCa3.1}$ and mitochondrial quality control in diabetic kidney disease is not yet known. Transforming growth factor $\beta 1$ (TGF- $\beta 1$ ) plays a central role in diabetic kidney disease. Recent studies indicate an emerging role of TGF- $\beta 1$ in the regulation of mitochondrial function. However, the molecular mechanism mediating mitochondrial quality control in response to TGF- $\beta 1$ remains limited. In this study, mitochondrial function was assessed in TGF- $\beta 1$-exposed renal proximal tubular epithelial cells (HK2 cells) transfected with scrambled siRNA or KCa3.1 siRNA. In vivo, diabetes was induced in KCa3.1+/+ and KCa3.1-/- mice by low-dose streptozotocin (STZ) injection. Mitochondrial fission/fusion-related proteins and mitophagy markers, as well as BCL2 interacting protein 3 (BNIP3) (a mitophagy regulator) were examined in HK2 cells and diabetic mice kidneys. The in vitro results showed that TGF- $\beta 1$ significantly inhibited mitochondrial ATP production rate and increased mitochondrial ROS (mtROS) production when compared to control, which was normalized by KCa3.1 gene silencing. Increased fission and suppressed fusion were found in both TGF- $\beta 1$-treated HK2 cells and diabetic mice, which were reversed by KCa3.1 deficiency. Furthermore, our results showed that mitophagy was inhibited in both in vitro and in vivo models of diabetic kidney disease. KCa3.1 deficiency restored abnormal mitophagy by inhibiting BNIP3 expression in TGF- $\beta 1$-induced HK2 cells as well as in the diabetic mice. Collectively, these results indicate that $\mathrm{KCa} 3.1$ mediates the dysregulation of mitochondrial quality control in diabetic kidney disease.

Keywords: diabetic kidney disease, mitochondrial quality control, mitochondrial dynamics, mitophagy, transforming growth factor $\beta 1, \mathrm{KCa} 3.1$ 


\section{INTRODUCTION}

Mitochondria are responsible for the main site of adenosine triphosphate (ATP) synthesis via oxidative phosphorylation (Alpers and Hudkins, 2011). Mitochondria have also been shown to play a crucial role in calcium signaling, reactive oxygen species (ROS) generation, apoptosis, necrosis, and innate immunity (Galluzzi et al., 2012; Suarez-Rivero et al., 2016). Mitochondrial dysfunction is characterized by a decrease in ATP production and increase in ROS generation leading to oxidative stress (Suarez-Rivero et al., 2016). Hence, maintaining optimal function of the mitochondria is important for maintaining cell survival, regulating cell death and cellular metabolic homeostasis (Sharma et al., 2003).

Mitochondrial quality control is exquisitely regulated to maintain functional mitochondria (Sharma et al., 2003). Mitochondrial quality control mechanisms are mainly regulated by mitochondrial dynamics and mitophagy (Ranjit et al., 2016). Mitochondrial dynamics include fission and fusion to repair or delete damaged components of the mitochondria. Mitochondrial fission allows for the segregation of damaged mitochondria, while mitochondrial fusion facilitates the exchanging of material between healthy mitochondria. Imbalanced mitochondrial fission and fusion are detrimental to mitochondrial function and cellular survival. Mitochondrial dynamics are regulated by several different GTPase proteins. Mitochondrial fission is regulated by dynamin-related protein 1 (Drp1) and mitochondrial fission protein 1 (Fis1). Mitochondrial fusion is mediated by mitofusin 1 (Mfn1), mitofusin 2 (Mfn2), and optic atrophy 1 (Opa1) proteins. Mfn1 and Mfn2 are localized on the mitochondrial outer membrane (MOM) and mediate tethering of MOM of adjacent mitochondria to promote the fusion of MOM, whereas Opa1 is responsible for mitochondrial inner membrane (MIM) fusion (Anand et al., 2014). Abnormalities in these mitochondrial dynamic proteins lead to severely altered mitochondrial morphology, defective mitochondrial function, and eventually cell death (Zhan et al., 2013). Mitophagy is selective autophagy to degrade and recycle dysfunctional or damaged mitochondria. Recent studies suggest that mitochondrial priming is mediated either through the Pink1/Parkin signaling pathway or the mitophagic receptors such as BCL2 interacting protein 3 (BNIP3), BNIP3 like (BNIP3L/NIX), and FUN14 domain containing 1 (FUNDC1) (Li et al., 2018; Wang J. et al., 2020). Disruption of mitochondrial networks prevents the elimination of damaged mitochondria and exacerbates ATP deficits, which is then implicated in a variety of diseases including diabetic kidney disease (Forbes and Thorburn, 2018; Suomalainen and Battersby, 2018). Although dysfunctional mitochondria are increasingly recognized to be central to the pathogenesis of diabetic kidney disease (Saxena et al., 2019), the understanding of the mechanism of mitochondrial quality control and its regulatory signaling pathways in diabetic kidney disease remains limited.

KCa3.1 (also known as IK1, SK4, or KCNN4) belongs to the calcium-activated potassium channel (KCa) family, which is localized in the plasma membrane, nucleus, and inner mitochondrial membranes (De Marchi et al., 2009; Chachi et al., 2013). KCa3.1 channels regulate calcium entry into cells through modulating calcium-signaling processes, which is necessary for maintaining various cellular activation processes such as proliferation, migration, and cytokine production (Cruse et al., 2006; Wulff and Castle, 2010; Chen et al., 2011). Hence, KCa3.1 has been proposed as a potential therapeutic target for sickle cell anemia, autoimmunity, and atherosclerosis (Wulff et al., 2007; Chou et al., 2008; Wulff and Castle, 2010). Recently, we have demonstrated an important role of KCa3.1 in diabetic kidney disease. Our studies have demonstrated that blockade of KCa3.1 alleviated renal fibrosis and inflammation in diabetic mice through inhibition of the TGF- $\beta 1$ signaling pathway and fibroblast activation (Huang et al., 2013, 2014b). Furthermore, our results showed that blockade of KCa3.1 is likely to exert its anti-fibrotic effects through the restoration of dysregulated tubular autophagy (Huang et al., 2016a). However, the mechanism by which KCa3.1 mediates mitochondrial quality control in diabetic kidney disease remains unknown.

It is well accepted that transforming growth factor $\beta 1$ (TGF- $\beta 1$ ) plays a central role in the development of diabetic kidney disease. Recent observations indicate an emerging role of TGF- $\beta 1$ in the regulation of mitochondrial function (Pozdzik et al., 2016; Choi et al., 2019). However, the molecular mechanism mediating mitochondrial quality control in response to TGF- $\beta 1$ remains limited. In this study, we investigated the effect of KCa3.1 silencing on mitochondrial function in TGF- $\beta 1$ stimulated human renal proximal tubular cells. We also assessed the role of KCa3.1 in mitochondrial dynamics and mitophagy as well as the underlying signaling pathways in both in vitro and in vivo models. Our results demonstrated that KCa3.1 deficiency was able to reverse diabetes-induced mitochondrial dysfunction by normalizing the disrupted mitochondrial quality control, which was likely mediated through inhibition of BNIP3 expression.

\section{MATERIALS AND METHODS}

\section{Materials}

Tissue culture medium and Lipofectamine 2000 were provided from Invitrogen Life Technologies (Carlsbad, CA, United States). Anti-LC3, anti-P62, anti-Cox4, anti-Mfn2, and anti-BNIP3 antibodies were purchased from Abcam (Cambridge, MA, United States), and anti- $\alpha$-tubulin antibody was from Sigma (St. Louis, MO, United States). Anti-phospho-Drp1 and horseradish peroxidase-conjugated secondary antibodies were purchased from Cell Signaling Technology (Danvers, MA, United States). Anti-Fis1 antibody was purchased from Proteintech (Rosemont, IL, United States), and Anti-Opal antibody was purchased from Novus Biologicals (Centennial, CO, United States). Alexa Fluor 488-conjugated secondary antibodies were obtained from Invitrogen (Carlsbad, CA, United States).

\section{Animal Studies}

Male $\mathrm{KCa} 3.1+/+$ mice and $\mathrm{KCa} 3.1-/-$ mice (6-8 weeks old) weighing approximately $20-25 \mathrm{~g}$ were used in the study. Mice were intraperitoneally injected with either $55 \mathrm{mg} / \mathrm{kg}$ of STZ (Sigma, St. Louis, MO, United States) diluted in $0.1 \mathrm{M}$ citrate buffer, $\mathrm{pH} 4.5$, or citrate buffer alone as described previously 
(Huang et al., 2013). Mice were weighed, and blood glucose level was determined using the Accu-chek glucometer (Roche Diagnostics). Mice with blood glucose greater than $16 \mathrm{mmol} / \mathrm{l}$ were considered to have diabetes.

This study was approved by the Animal Research Ethics Committee of Royal North Shore Hospital (1101-001A). Experimental procedures adhered to the guidelines of the National Health and Medical Research Council of Australia's Code for the Care and Use of Animals for Scientific Purposes.

\section{Cell Culture and KCa3.1 Gene Silencing}

Immortalized human renal proximal tubular cells (HK2 cells), obtained from ATCC (Manassas, VA, United States), were grown in keratinocyte serum-free media (Invitrogen, Carlsbad, CA, United States). All experiments were performed at passages 5-15.

HK2 cells were transfected with either KCa3.1 siRNA or scrambled control siRNA using Lipofectamine 2000 reagent (Invitrogen, Carlsbad, CA, United States) according to the manufacturer's instruction. The transfected cells were then incubated with TGF- $\beta 1(2 \mathrm{ng} / \mathrm{ml})$ for $48 \mathrm{~h}$. The siRNA sequence for KCa3.1 is $5^{\prime}$-GCACCUUUCAGACACACUU-3' (GenePharma, Shanghai).

\section{Mitochondrial ATP Production Rate}

Mitochondrial ATP production rate was determined using the ATP bioluminescence assay kit (Roche Diagnostics, Switzerland) according to the protocol described previously (Huang et al., 2016b). ATP production was induced by incubation of the cell suspension with substrate buffer at $37^{\circ} \mathrm{C}$ for $10 \mathrm{~min}$, which was then stopped by addition of boiling quenching buffer at $100^{\circ} \mathrm{C}$ for $2 \mathrm{~min}$. The reaction mixture diluted 1:10 in quenching buffer was measured using an FB10 luminometer (Berthold Detection Systems, Germany) to determine the ATP level.

\section{Mitochondrial Superoxide Quantification}

Mitochondrial superoxide was detected by MitoSOX Red staining (Molecular Probes-Invitrogen) as described previously (Li et al., 2019). Briefly, the treated cells were incubated with $5 \mu \mathrm{M}$ MitoSOX Red for $15 \mathrm{~min}$ at $37^{\circ} \mathrm{C}$. After washing with warm buffer, the stained cells were then visualized under confocal fluorescence microscopy (Leica Microsystems, Mannheim, Germany). The results were expressed as the fluorescence intensity normalized to the control group.

\section{Transmission Electron Microscopy}

The cell samples were prepared for transmission electron microscopic analysis as previously reported (Huang et al., 2014a). Briefly, after washing with pre-warmed PBS, the cells were next fixed in $2 \%$ glutaraldehyde for $1 \mathrm{~h}$. Subsequently, the fixed cells were postfixed with $1 \%$ osmium tetroxide for $1 \mathrm{~h}$ after briefly washing with PBS. The samples were rinsed in distilled water, stained with $1 \%$ tannic acid, dehydrated in a gradient of ethanol, and embedded in Epon. Sections of $70 \mathrm{~nm}$ were generated with an ultramicrotome (Ultracut 7, Leica) and poststained with $2 \%$ aqueous uranyl acetate and Reynold's lead citrate for $10 \mathrm{~min}$ each. The specimens were examined under a transmission electron microscope operating at $200 \mathrm{kV}$ (JEM2100, JEOL, Japan). Mitochondrial Feret's diameter (maximum and minimum), the distance between two parallel tangential lines within the selected mitochondrion, was determined using Image J (Demeter-Haludka et al., 2018; Lomash et al., 2019).

\section{Immunocytofluorescence Staining}

To monitor mitophagy, HK2 cells were stained with $1 \mathrm{nM}$ of MitoTracker Deep Red FM for $15 \mathrm{~min}$ at $37^{\circ} \mathrm{C}$ (Huang et al., 2016b). After fixation and blocking, the cells were incubated with primary antibodies against LC3 or P62 in 2\% BSA in PBS for $1 \mathrm{~h}$, followed with Alexa Fluor-488 conjugated secondary antibodies for $40 \mathrm{~min}$. The cells were then counterstained and mounted with 4',6-diamidino-2 phenylindole (DAPI)mounting medium (Invitrogen). The fluorescent signals were collected and analyzed by confocal fluorescence microscopy (Leica Microsystems, Mannheim, Germany).

\section{Mitochondrial Isolation}

Mitochondria were isolated from mice renal cortex as described previously (Nguyen et al., 2015). Briefly, tissue samples were homogenized in HEPES buffer $(20 \mathrm{mM}$, pH 7.2, containing $1 \mathrm{mM}$ EGTA, $210 \mathrm{mM}$ mannitol, and $70 \mathrm{mM}$ sucrose). The homogenate was centrifuged at $1,500 \times g$ for $5 \mathrm{~min}$ at $4^{\circ} \mathrm{C}$. The supernatant was collected and then centrifuged at $10,000 \times g$ for $15 \mathrm{~min}$ at $4^{\circ} \mathrm{C}$ to pellet the mitochondria, which were resuspended in HEPES buffer for analyses. The supernatants were collected as the cytosol fraction. The total protein concentration of the isolated mitochondrial and cytosol fraction was determined by the BCA Protein Assay Kit (Thermo Scientific).

\section{Western Blotting}

An equal amount of cell and tissue lysate samples was separated by SDS-PAGE, and then transferred to Hybond ECL nitrocellulose membrane (Amersham, United States). The membranes were blocked and then probed with primary antibodies (LC3, P62, p-Drp1, Fis1, Opa1, Mfn2, Cox4, BNIP3, and $\alpha$-Tubulin) at $4^{\circ} \mathrm{C}$ overnight followed with HRP-conjugated secondary antibody (Amersham, United States). The membrane blots were detected and quantified using LAS-4000 Imaging System (FUJIFILM, Japan).

\section{Statistical Analysis}

The data were expressed as mean \pm SEM. Statistical analysis between two groups was evaluated by two-tailed $t$-test. Comparison of the results from multiple groups was performed by one-way ANOVA, followed by Tukey post-test. A Pvalue $<0.05$ was considered as statistically significant.

\section{RESULTS}

\section{KCa3.1 Gene Silencing Reversed TGF- $\beta 1$-Induced Mitochondrial Dysfunction in HK2 Cells}

To determine the role of $\mathrm{KCa} 3.1$ in mitochondrial function, mitochondrial ATP production rate was first examined in 
HK2 cells exposed to TGF- $\beta 1$ with or without KCa3.1 siRNA. As shown in Figure 1A, compared to the controls, TGF$\beta 1$ significantly inhibited mitochondrial ATP production rate in $\mathrm{HK} 2$ cells transfected with scrambled siRNA $(19.08 \pm 0.82$ for the control and $12.77 \pm 0.56$ for TGF$\beta 1+$ scrambled siRNA, $P<0.01$, Figure 1A). Inhibition of KCa3.1 with KCa3.1 siRNA reversed TGF- $\beta 1$-induced inhibition of ATP production rate $(15.84 \pm 0.29, P<0.01$, Figure 1A).

Mitochondrial ROS (mtROS) production in HK2 cells was then examined by fluorescence staining with MitoSOX Red, which is designed for highly selective detection of superoxide in mitochondria. As shown in Figure 1B, a low level of fluorescence was found in the control cells, indicating normal basal levels of mtROS production. Compared to the control cells, TGF- $\beta 1$ induced increased mtROS production, characterized by the elevated fluorescent intensity of MitoSOX in $\mathrm{HK} 2$ cells. KCa3.1 gene silencing significantly reduced TGF- $\beta 1$-induced mtROS generation $(P<0.01$, Figure 1C). These data collectively demonstrate that TGF- $\beta 1$ impaired mitochondrial function through a KCa3.1-related mechanism in HK2 cells and KCa3.1 gene silencing reversed TGF- $\beta 1$-induced mitochondrial dysfunction.

\section{KCa3.1 Gene Silencing Attenuated TGF- $\beta 1$-Induced Increased Fission and Suppressed Fusion in HK2 Cells}

To determine whether KCa3.1 has any effect on mitochondrial fission and fusion processes, mitochondrial fission-related protein Drp1, Fis1, and mitochondrial fusion-related protein Opa1, Mfn2 were examined in HK2 cells exposed to TGF$\beta 1$ with or without KCa3.1 gene silencing. As shown in Figure 2A, TGF- $\beta 1$ significantly increased the level of profission protein Drp1 expression compared to the control group $(P<0.01)$. This increase was attenuated by KCa3.1 gene silencing $(P<0.05$, Figure $2 \mathrm{~A})$. In response to TGF- $\beta 1$, the levels of profusion protein Opa1 expression in HK2 cells were significantly decreased compared to the control group $(P<0.05$, Figure 2C), which was attenuated by $\mathrm{KCa} 3.1$ gene silencing $(P<0.05$, Figure 2C). Interestingly, the expression of Fis 1 and Mfn2 was not obviously altered by TGF- $\beta 1$ stimulation (Figures 2B,D).

\section{KCa3.1 Gene Silencing Reversed TGF- $\beta 1$-Induced Inhibition of Mitophagy}

The mitochondrial shape is maintained through the processes of mitochondrial fission and fusion (Zhang et al., 2019). To investigate the role of KCa3.1 in mitochondrial morphology, we employed transmission electron microscopy to assess the fine structure of mitochondria at high resolution. The control group cells exhibited healthy, normal appearing mitochondria with well-developed cristae (Figure 3A). In contrast, an abundance of mitochondria with severely disrupted cristae was found in HK2 cells exposed to TGF- $\beta 1$, which was attenuated by KCa3.1 gene silencing. As shown in Figures 3B,C, compared to the control group, exposure to
TGF- $\beta 1$ resulted in a significant reduction in maximum and minimum Feret's diameter of the mitochondria, indicating that the mitochondria became smaller following the TGF$\beta 1$ insult. These alterations were significantly recovered by KCa3.1 gene silencing.

Mitochondrial autophagy was further studied by colocalization of autophagy markers LC3 and P62 with MitoTracker Deep Red stained mitochondria. As shown in Figures 3D,E, the intensity of LC3 that colocalized with MitoTracker Deep Red stained mitochondria was significantly increased in HK2 cells exposed to TGF- $\beta 1$ when compared to the control $(P<0.01$, Figure 3E), which was significantly attenuated by KCa3.1 gene silencing $(P<0.05$, Figures 3D,E). Similarly, exposure of cells to KCa3.1 siRNA significantly suppressed TGF- $\beta 1$-induced increased intensity of P62 colocalized with the mitochondria $(P<0.05$, Figures 3F,G). These data indicate that KCa3.1 gene silencing reversed TGF- $\beta 1$-induced inhibition of mitophagy in HK2 cells.

\section{KCa3.1 Deficiency Attenuated Diabetes-Induced Increased Fission and Suppressed Fusion in Diabetic Mice}

To further confirm the effect of KCa3.1 on diabetes-related mitochondrial dynamics, mitochondrial fission- and fusionrelated proteins were assessed in mice kidneys. As shown in Figure 4, diabetes significantly increased the level of pro-fission protein Drp1 and suppressed the level of profusion protein Opa1 in diabetic KCa3.1 wild-type mice $(\mathrm{K}+/+$ $\mathrm{DM})$ compared to non-diabetic control mice $(\mathrm{K}+/+$ control) $(P<0.05$, Figures 4A,C). However, the changes were attenuated in diabetic KCa3.1 deficient mice $(\mathrm{K}-/-\mathrm{DM})(P<0.05$, Figures 4A,C). Conversely, the levels of Fis1 and Mfn2 were not notably changed in mice kidneys (Figures 4B,D). The findings suggested that KCa3.1 regulates diabetes-induced imbalance in mitochondrial dynamics by enhancing fission and reducing fusion.

\section{KCa3.1 Deficiency Attenuated Diabetes-Induced Inhibition of Mitophagy in Diabetic Mice}

To determine whether KCa3.1 deficiency attenuates diabetic renal fibrosis via regulating mitophagy, the autophagy markers LC3 and P62 were assessed in mitochondria from diabetic kidney tissues using western blot analysis. As shown in Figure 5A, increased expression of LC3 in mitochondria was observed in diabetic KCa3.1 wild-type mice $(\mathrm{K}+/+\mathrm{DM})$ when compared to the non-diabetic controls $(\mathrm{K}+/+$ control $)(P<0.05)$. KCa3.1 deficiency significantly attenuated diabetes-induced upregulation of LC3 expression in mitochondria from diabetic KCa3.1 deficient mice $(\mathrm{K}-/-\mathrm{DM})(P<0.05$, Figure 5A). In line with the LC3 findings, western blot analysis results showed that P62 expression in mitochondria was significantly increased in diabetic kidneys as compared to the non-diabetic controls $(P<0.05$, Figure 5B), which was inhibited in diabetic KCa3.1 deficient mice $(P<0.05$, Figure 5B). Collectively, these results indicate 
A

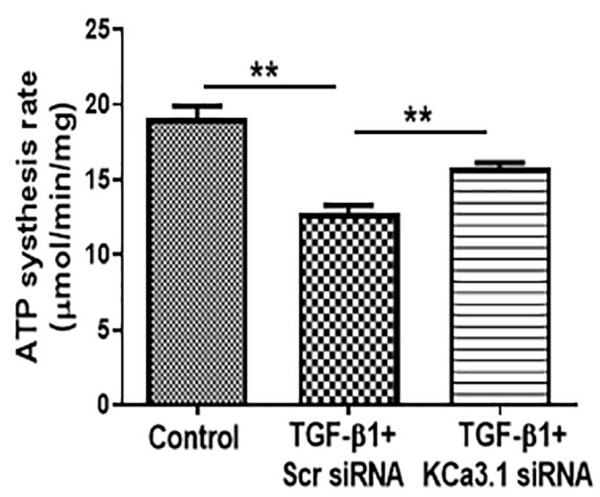

B Control

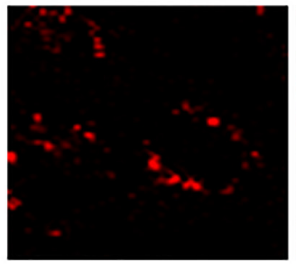

TGF- $\beta 1+$ Scr SIRNA

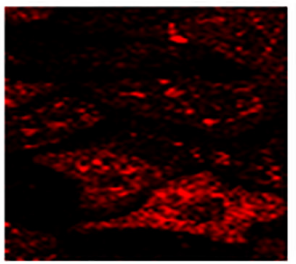

TGF- $\beta 1+$

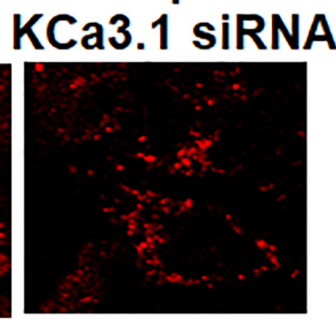

C

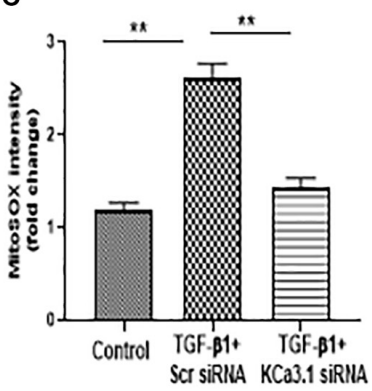

FIGURE 1 | KCa3.1 gene silencing reversed TGF- $\beta 1$-induced mitochondrial dysfunction in HK2 cells. (A) Mitochondrial ATP production rate was assessed to detect mitochondrial function. KCa3.1 silencing significantly increased TGF- $\beta 1$-induced inhibition of ATP production rate. (B) Mitochondrial reactive oxygen species (mtROS) production was assessed by MitoSOX Red staining. KCa3.1 silencing significantly reduced TGF- $\beta 1$-induced mtROS overproduction. (C) Quantification of MitoSOX Red fluorescence intensity normalized to the control group in HK2 cells. Results are presented as mean \pm SEM. ${ }^{\star \star} P<0.01$. $N=3$. Original magnification: $\times 600$.

that KCa3.1 deficiency attenuates diabetes-induced inhibition of mitophagy in diabetic mice.

\section{KCa3.1 Deficiency Suppressed Diabetes-Induced Upregulation of BNIP3 Expression in HK2 Cells and Diabetic Mice}

To investigate the mechanism whereby $\mathrm{KCa} 3.1$ regulates mitophagy, BNIP3, a regulator of mitophagy, was examined in HK2 cells exposed to TGF- $\beta 1$ as well as diabetic mice kidneys. As shown in Figure $\mathbf{6 A}$, the expression of BNIP3 was significantly increased by TGF- $\beta 1$ in HK2 cells $(P<0.05$, Figure 6A), which was attenuated by $\mathrm{KCa} 3.1$ gene silencing $(P<0.01$, Figure 6A). Similarly, the western blot analysis confirmed a marked induction of BNIP3 in diabetic KCa3.1 wildtype mice $(\mathrm{K}+/+\mathrm{DM})$ when compared to non-diabetic control mice $(\mathrm{K}+/+$ control $)(P<0.05$, Figure 6B). KCa3.1 deficiency significantly attenuated diabetes-induced upregulation of BNIP3 expression in diabetic KCa3.1-/ - mice $(\mathrm{K}-/-\mathrm{DM})(P<0.05$, Figure 6B). Together, these results suggest that KCa3.1-mediated dysregulation of mitophagy is associated with upregulation of BNIP3 expression.

\section{DISCUSSION}

This study was undertaken to define the role of KCa3.1 in regulating mitochondrial quality control in diabetic kidney disease as depicted in Figure 7. The study demonstrated that TGF- $\beta 1$ resulted in mitochondrial dysfunction and subsequent mtROS overproduction as well as inhibition of mitophagy, which leads to the disruption of the mitochondrial quality control, eventually causing tubular cell injury. KCa3.1 deficiency restored abnormal mitochondrial dysfunction and mitochondrial quality control by improving BNIP3-mediated mitophagy in TGF- $\beta 1$-induced renal proximal tubular cells as well as in STZinduced diabetic mice.

Transforming growth factor $\beta 1$, the most abundant isoform of TGF- $\beta$ family members, can be secreted by all types of renal cells and infiltrating inflammatory cells. It is well established that TGF- $\beta 1$ acts as a pivotal mediator in diabetic kidney 

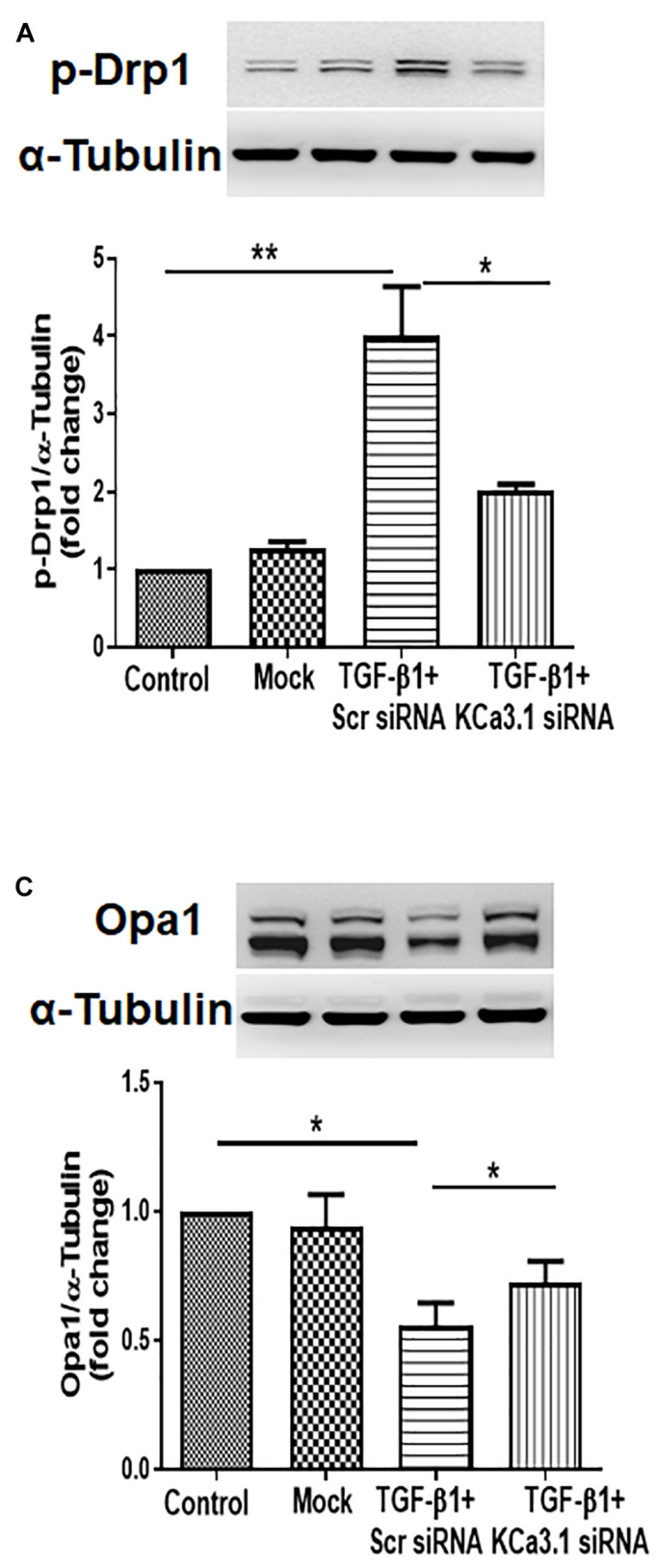
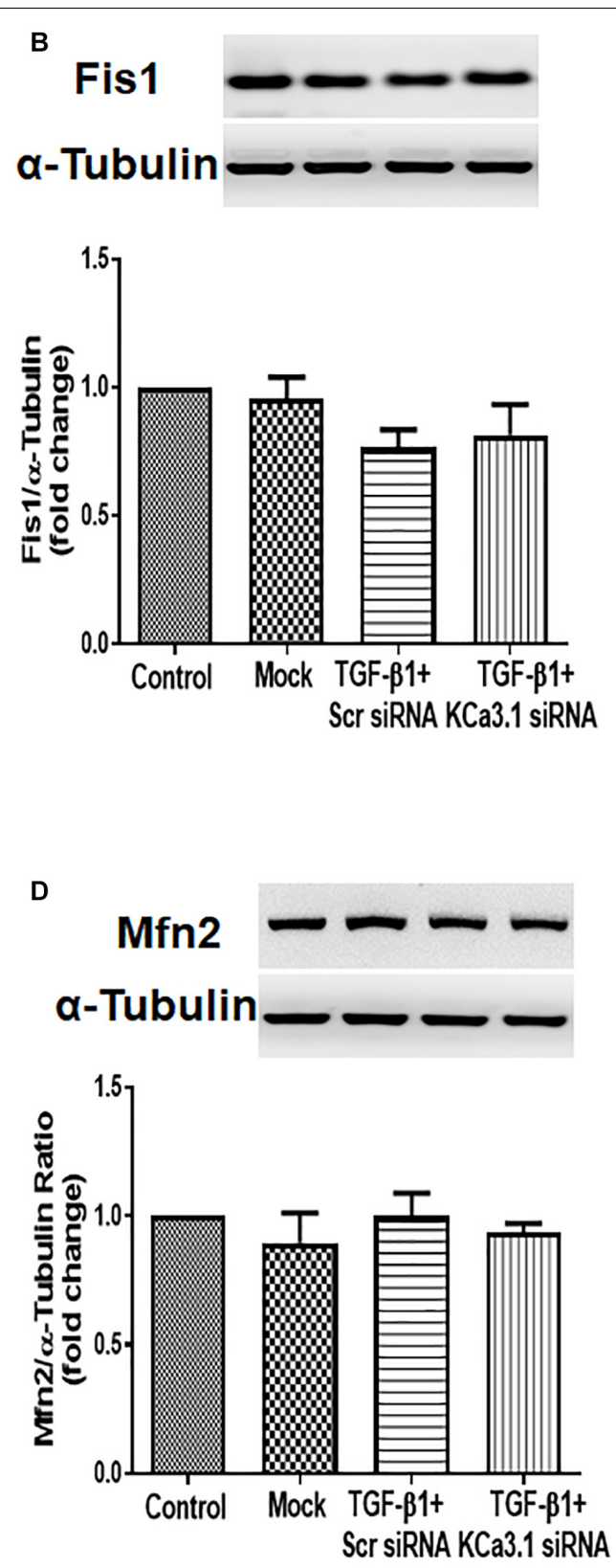

FIGURE 2 | KCa3.1 gene silencing attenuated TGF- $\beta 1$-induced increased fission and suppressed fusion in HK2 cells. Mitochondrial pro-fission proteins (Drp1 and Fis1) and pro-fusion mediators (Opa1 and Mfn2) were examined by western blotting. Western blot analyses revealed an increased expression of Drp1 (A) and a reduced expression of Opa1 (C) in TGF- $\beta$ 1-induced HK2 cells, which were reversed by KCa3.1 gene silencing. There were no changes in the expression of Fis1 (B) and Mfn2 under TGF- $\beta 1$ stimulation (D). Results are presented as mean \pm SEM. ${ }^{\star} P<0.05,{ }^{\star \star} P<0.01, N=3$.

disease given its involvement in renal fibrosis, inflammation, cell growth, apoptosis, and differentiation (Bottinger, 2007; Hills and Squires, 2011; Lan, 2012; Meng et al., 2013). A growing body of evidence indicates that mitochondrial dysfunction may be important in the development and progression of diabetic kidney disease (Forbes and Thorburn, 2018; Saxena et al., 2019). Recent studies have revealed a link between TGF- $\beta 1$ and mitochondrial dysfunction. In vitro studies demonstrated that TGF- $\beta 1$-induced mitochondria dysfunction has been found in various types of cells including lung epithelial cells (Patel et al., 2015), alveolar macrophages (Grunwell et al., 2018), and subepithelial fibroblasts (Sun et al., 2019) as well as renal cells (Yu et al., 2016; Wang Y. et al., 2020). Yu et al. (2016) reported that a TGF- $\beta 1$-induced fibrotic phenotype was associated with significant mitochondrial dysfunction in mouse renal tubular cells, which was markedly improved by MnTBAP (a cell-permeable mimic of superoxide dismutase) treatment. Recently, mitochondrial dysfunction was found in rat kidney fibroblast cells under TGF- $\beta 1$ challenge 


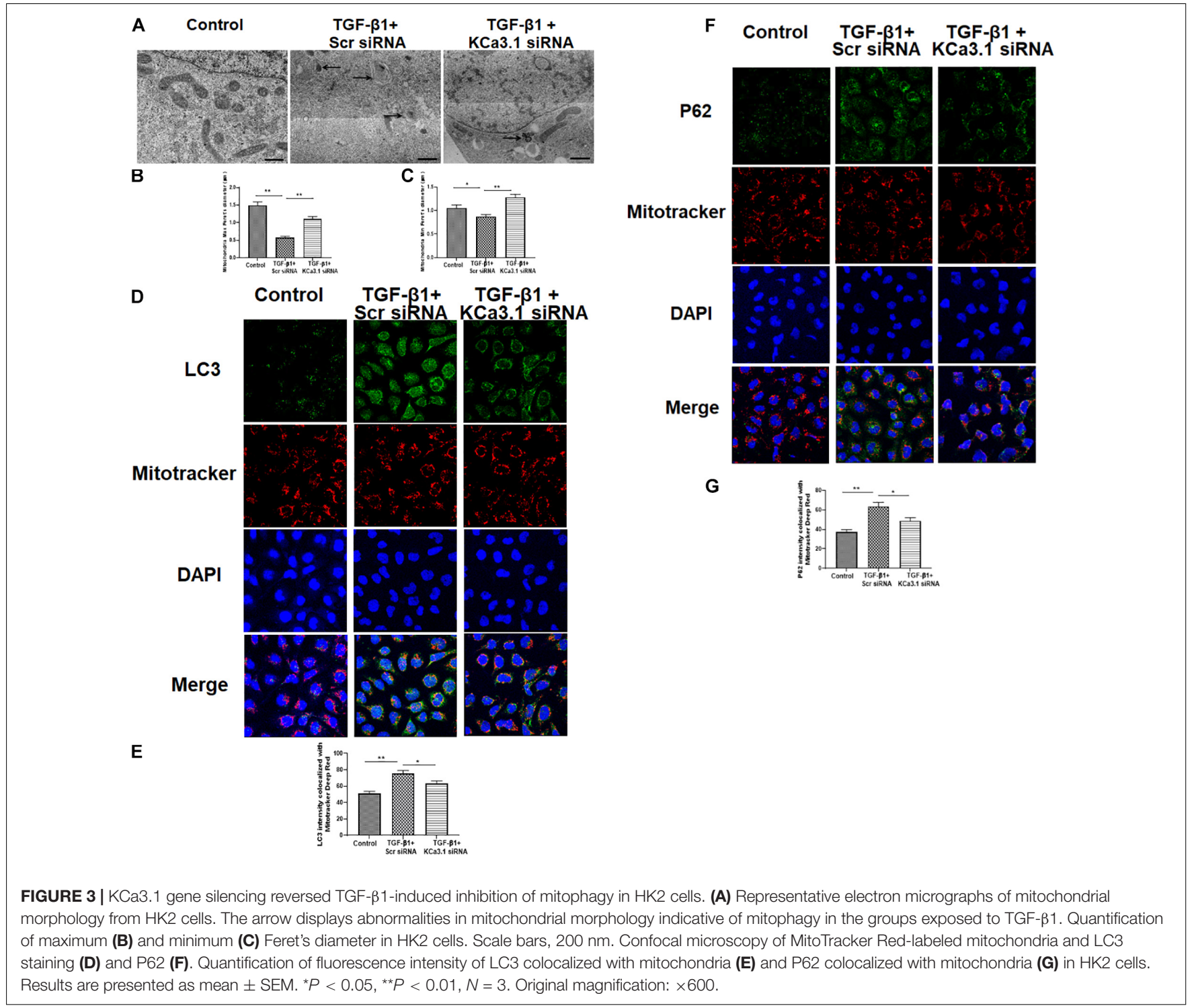

together with fibroblast activation (Wang Y. et al., 2020). Similarly, in vivo studies have also demonstrated that increasing TGF- $\beta 1$ activity is associated with mitochondrial dysfunction and increasing mtROS synthesis in various diseases including diabetic kidney disease (Lee et al., 2017). In line with previous studies, the interaction between TGF- $\beta 1$ signaling and mitochondria has been demonstrated in the current study. Our previous study showed that the anti-fibrotic effect of KCa3.1 inhibition was likely mediated by antagonizing TGF- $\beta 1$ signaling through suppression of TGF- $\beta 1$ and TGF- $\beta$ receptor II expression and the downstream Smad2/3 pathway in diabetic kidney disease (Huang et al., 2013). Our current results show that TGF- $\beta 1$ induces mitochondrial dysfunction, as indicated by suppressed ATP production and increased mtROS production in renal proximal tubular cells (Figure 1). Furthermore, our results demonstrate that TGF- $\beta 1$ exposure leads to altered mitochondrial morphology and increased accumulation of LC3 and P62 colocalized with mitochondria by immunofluorescence staining in renal proximal tubular cells, suggesting that TGF- $\beta 1$ impaired mitochondrial function and mitophagy flux in renal tubular cells (Figure 3). Taken together, our previous and current studies demonstrate that activation of the TGF- $\beta 1$ signaling pathway (Huang et al., 2013) and mitochondrial dysfunction are both recovered by KCa3.1 deficiency, indicating that improving mitochondrial function may be a key mechanism by which inhibition of KCa3.1 protects the kidney from diabetes-induced fibrosis.

Mitochondria are dynamic organelles that are constantly undergoing fission and fusion to repair damaged components of the mitochondria and maintain the homeostasis of cells. During mitochondrial fission, Drp1 is recruited from the cytosol onto the MOMs to interact with various receptors, such as Fis1, mitochondrial fission factor (MFF), and mitochondrial dynamic proteins of 49 and $51 \mathrm{kDa}$ (MiD49 and MiD51). Opa1, a dynamin protein, is involved in mitochondrial fusion, 
A

\section{p-Drp1}

$\alpha$-Tubulin

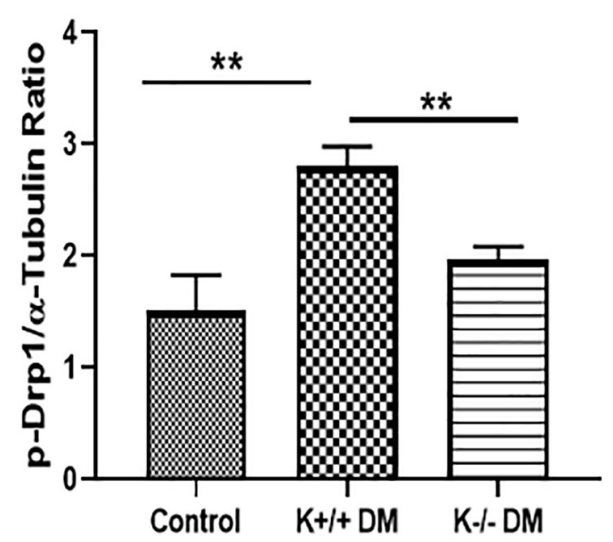

C

Opa1

$\alpha-T u b u l i n$

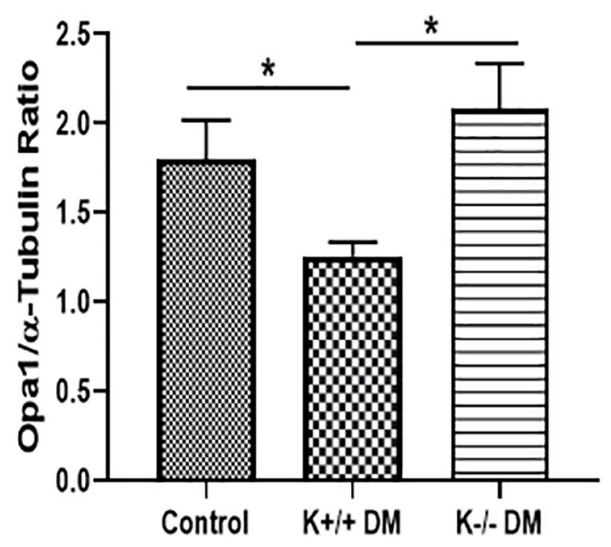

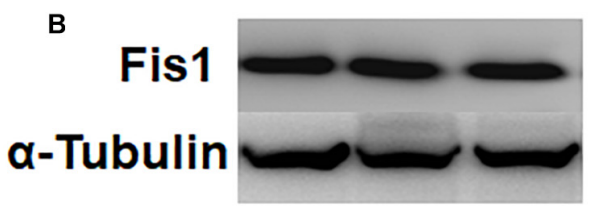

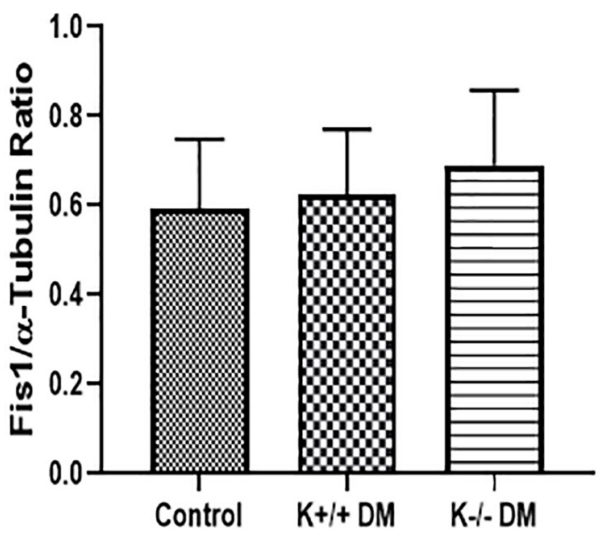

D
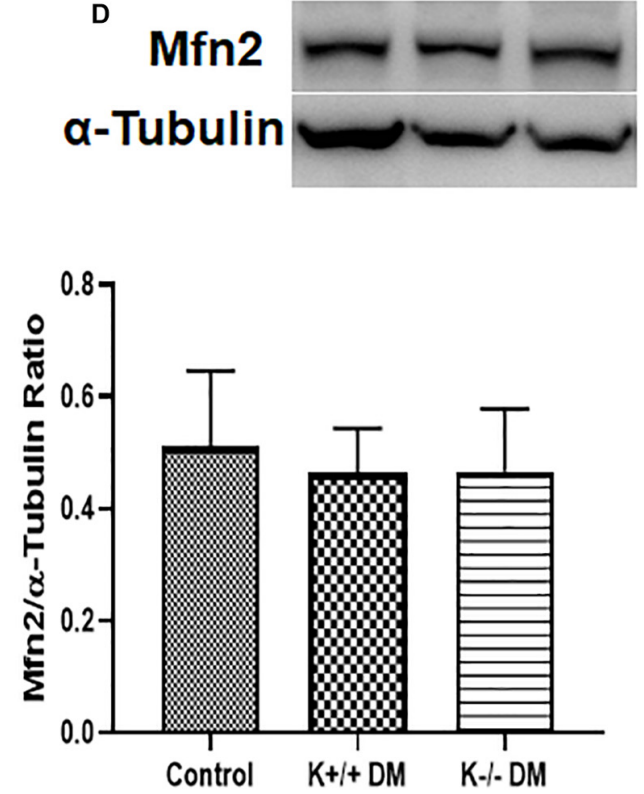

FIGURE 4 | KCa3.1 deficiency attenuated diabetes-induced increased fission and suppressed fusion in diabetic mice. Mitochondrial pro-fission proteins (Drp1 and Fis1) and pro-fusion mediators (Opa1 and Mfn2) were examined by western blotting in kidney tissues. Western blot analysis revealed an increased expression of Drp1 (A) and a reduced expression of Opa1 (C) in diabetic KCa3.1+/+ mice, which were reversed in KCa3.1 deficient mice (K-/- DM). The levels of Fis1 (B) and Mfn2 (D) were not notably changed in mice kidneys. Results are presented as mean $\pm \mathrm{SEM}$. ${ }^{\star} P<0.05,{ }^{\star \star} P<0.01, N=5$.

cristae structure maintenance, and apoptosis (Del Dotto et al., 2018). Opa1 has eight alternatively spliced isoforms, which can be further processed by proteases yeast mitochondrial escape 1 like 1 ATPase and metalloendopeptidase OMA1 to convert the long Opal (L-Opa1) into a cleaved short Opa1 (S-Opa1) form (Anand et al., 2014). L-Opal is competent for mitochondrial fusion, while the function of S-Opal is still not clear. However, both forms are essential for the 


\section{A LC3-I \\ LC3-II}

Cox4
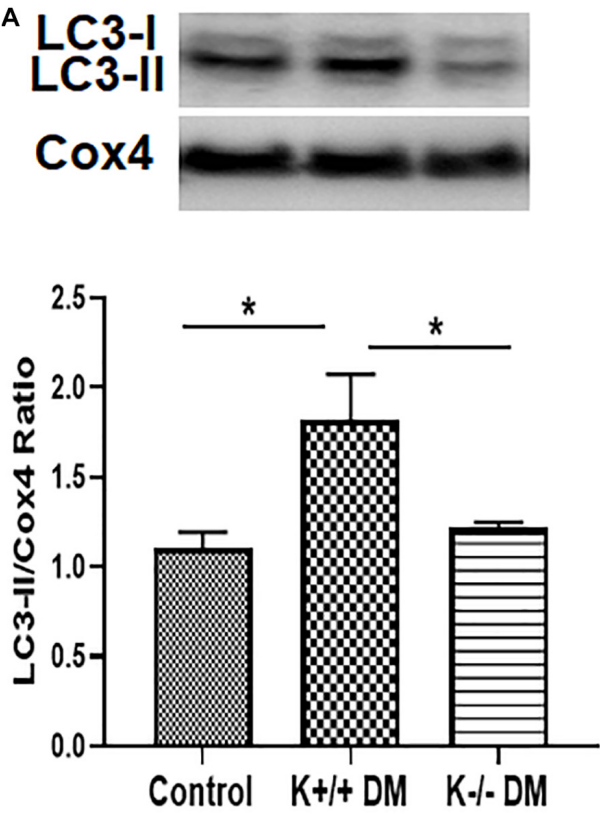

B

P62

Cox4
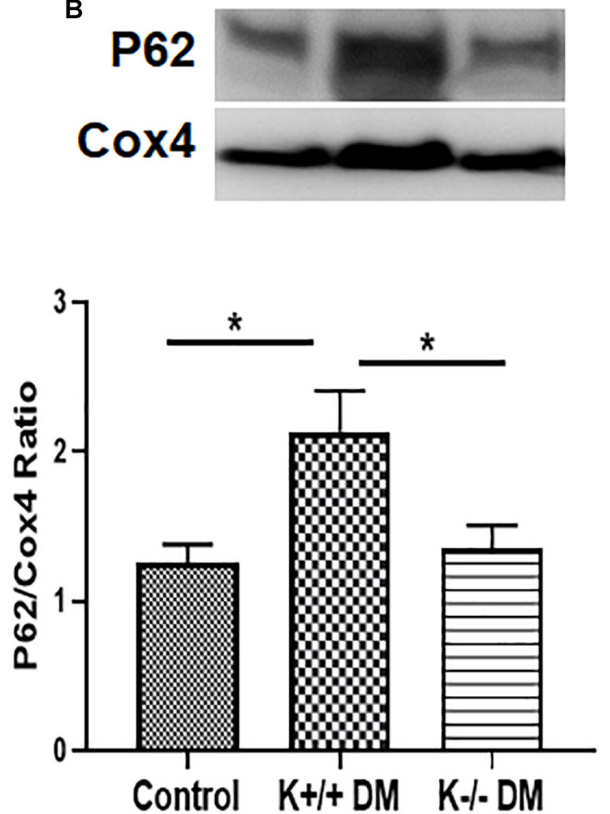

FIGURE 5 | KCa3.1 deficiency attenuated diabetes-induced inhibition of mitophagy in diabetic mice. The autophagy markers LC3 and P62 were assessed in mitochondria from diabetic kidney tissues using western blot analysis. Western blot analyses revealed an increased expression of LC3 (A) and P62 (B) in diabetic $\mathrm{KCa3} .1+/+$ mice, which were significantly attenuated in KCa3.1 deficient mice $(\mathrm{K}-/-\mathrm{DM})$. Results are presented as mean $+\mathrm{SEM}$. ${ }^{\star} P<0.05, N=5$.

\section{A \\ BNIP3 $\alpha$-Tubulin}

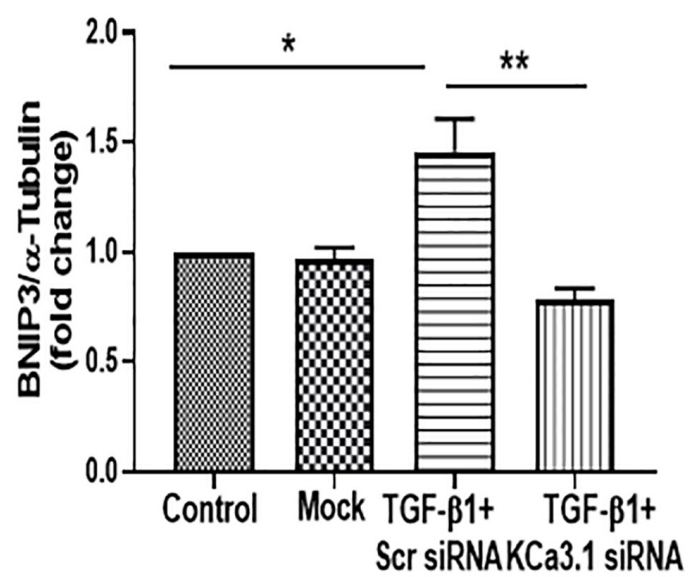

B

BNIP3

a-Tubulin
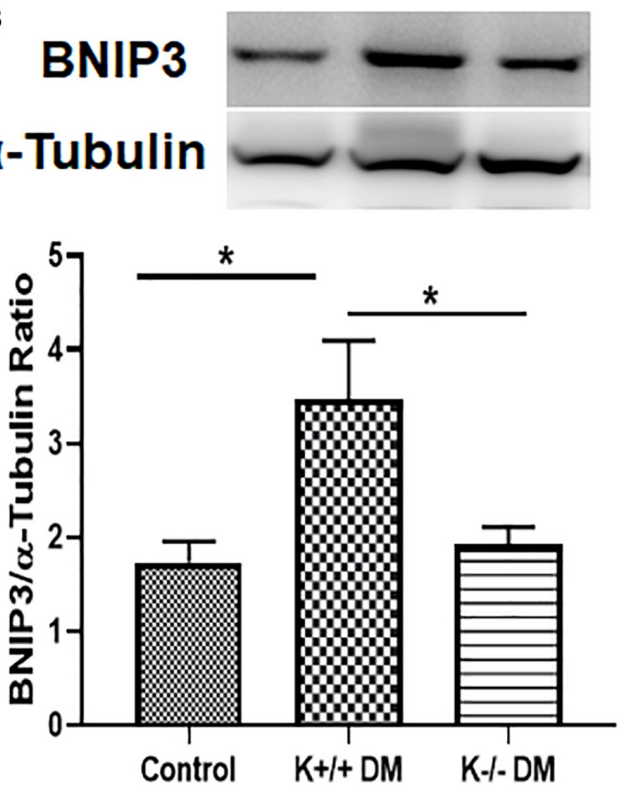

FIGURE 6 | KCa3.1 deficiency suppressed diabetes-induced upregulation of BNIP3 expression in HK2 cells and diabetic mice. (A) Western blot analysis showed that KCa3.1 silencing inhibited TGF- $\beta 1$-induced BNIP3 expression in HK2 cells. $N=3$. (B) Western blot analysis revealed an increased expression of BNIP3 in diabetic KCa3.1+/+ mice, which were significantly attenuated in KCa3.1 deficiency mice (K-/- DM). Results are presented as mean \pm SEM. ${ }^{*} P<0.05,{ }^{* *} P<0.01$, $N=5$.

function of Opal on mitochondrial dynamics and architecture (Del Dotto et al., 2018). The imbalance in mitochondrial fission and fusion largely contributes to tissue pathology in a variety of metabolic conditions, including kidney diseases (Sun et al., 2017; Cassina et al., 2020; Wang Y. et al., 2020; Zhang et al., 2020). Sun et al. (2017) showed expression of 


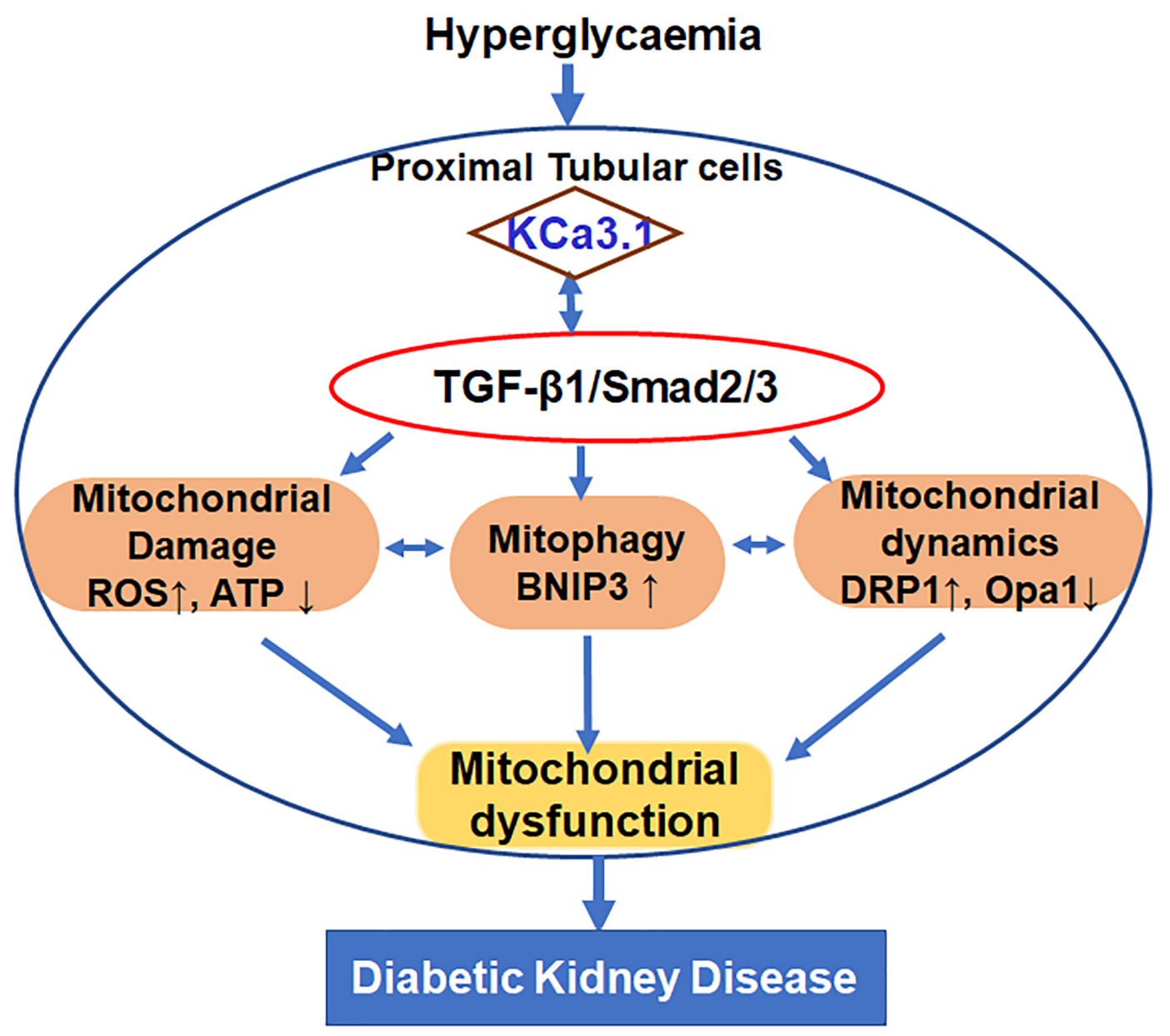

FIGURE 7 | Schematic diagram depicting the conceivable cellular events by which KCa3.1 mediates dysregulation of mitochondrial quality control in diabetic kidney disease.

the mitochondrial pro-fission protein DRP1 is increased and the mitochondrial pro-fusion protein Opal declined in 5/6 nephrectomized $(\mathrm{Nx})$ rats and TGF- $\beta 1$-exposed HK2 cells. In addition, increased expression of Drp1 and downregulation of Opal expression has been found in other animal models of kidney diseases including autosomal dominant polycystic kidney disease, obstructive nephropathy, and STZ-induced diabetic kidney disease (Cassina et al., 2020; Wang Y. et al., 2020; Zhang et al., 2020). Restoration of the imbalanced expression of all these mitochondrial dynamics-associated proteins has been proven to exert renoprotective effects. Consistently, in the present study, excessive mitochondrial fission and decreased fusion have also been demonstrated in TGF- $\beta$ 1-exposed HK2 cells and in kidneys of diabetic mice, as evidenced by upregulation of Drp1 and downregulation of Opa1 (Figures 2, 4). As expected, the long form of Opal was downregulated, suggesting decreased fusion. However, the conversion of the long form to the short form of Opal was not observed in our study, as indicated by a reduction in the short form of Opa1. The other protease systems such as the ubiquitin proteasome pathway may also be involved in the model of diabetic kidney disease, which deserves further investigation. In our study, KCa3.1 deficiency normalized the expression of mitochondrial dynamic proteins to mitigate the altered mitochondrial dynamics, suggesting that the anti-fibrotic effects of KCa3.1 inhibition may be partly attributed to the modulation of mitochondrial dynamics.

Mitophagy is a form of selective autophagy, which eliminates damaged or defective mitochondria. Recently, mitophagy has emerged as a cytoprotective mechanism to maintain mitochondrial homeostasis and cell survival under conditions of stress. Defective mitophagy has been reported in various kidney diseases including cisplatin-induced acute kidney injury (Zhao et al., 2017), ischemia-reperfusion-induced acute kidney injury (Ishihara et al., 2013), and diabetic kidney disease (Li et al., 2017; Xiao et al., 2017). Consistently, we found that mitophagy was markedly decreased in both in vitro and in vivo studies (Figures 3, 5), which was accompanied by mitochondrial dysfunction. BNIP3, a member of the $\mathrm{Bcl} 2$ family, has been identified as a key receptor for mitophagy via interaction with LC3 (Hanna et al., 2012). BNIP3 resides primarily on the mitochondria and is a critical regulator of 
mitochondrial function and cell apoptosis (Gao et al., 2020). Specifically, increasing BNIP3 expression leads to loss of mitochondrial membrane potential and the opening of the mitochondrial permeability transformation pore, which results in mitochondrial dysfunction and cell death (Kubli et al., 2007). BNIP3 has been shown to be involved in many diseases such as hepatic, cardiovascular diseases, and cancer (Kanzawa et al., 2005; Dhingra et al., 2017; Gong et al., 2018). In kidneys, Ishihara et al. (2013) observed the induction of BNIP3 together with increased apoptosis and defective autophagy/mitophagy under hypoxic conditions in renal tubular cells and in ischemia-reperfusion injury in rats. Tang et al. (2019) further demonstrated an important role of BNIP3-mediated mitophagy in mitochondrial quality control, tubular cell survival, and renal function during ischemia-reperfusion injury. Recently, Liu et al. (2019) reported that Stanniocalcin-1 ameliorates oxidative stress and cell apoptosis in the kidneys of the $\mathrm{db} / \mathrm{db}$ mice and high glucose-treated mouse proximal tubular cells by inhibiting BNIP3 expression, which is mediated by activating the AMPK/Sirt3 pathway. In our study, the increased BNIP3 expression was found to be related to dysfunctional mitochondria and abnormal mitochondrial dynamics in TGF- $\beta 1$-exposed HK2 cells and STZ induced type 1 diabetic mice (Figure 6). KCa3.1 deficiency restored mitochondrial quality surveillance by inhibiting BNIP3 expression, indicating a potential relationship between KCa3.1 and BNIP3. It is important to point out that other mitophagy-related pathways such as PINK1/Parkin and FUNDC1-dependent mitophagy have also been reported in diabetic kidney disease (Xiao et al., 2017; Liu et al., 2020; Wei et al., 2020), which were not examined in this study. Hence, further study is warranted to better understand the role and the interaction between different mitophagy-related pathways in diabetic kidney disease.

\section{CONCLUSION}

These studies in both in vitro and in vivo models demonstrate that KCa3.1 mediates dysregulation of mitochondrial function, mitochondrial dynamics, and mitophagy in diabetic kidney disease. Functional KCa3.1 has been shown to be expressed in the inner mitochondrial membrane in addition to the plasma membrane (Leanza et al., 2014; Kovalenko et al., 2016). Although the exact regulatory mechanism of $\mathrm{KCa} 3.1$

\section{REFERENCES}

Alpers, C. E., and Hudkins, K. L. (2011). Mouse models of diabetic nephropathy. Curr. Opin. Nephrol. Hypertens. 20, 278-284. doi: 10.1097/MNH. 0b013e3283451901

Anand, R., Wai, T., Baker, M. J., Kladt, N., Schauss, A. C., Rugarli, E., et al. (2014). The i-AAA protease YME1L and OMA1 cleave OPA1 to balance mitochondrial fusion and fission. J. Cell Biol. 204, 919-929. doi: 10.1083/jcb.201308006

Bottinger, E. P. (2007). TGF-beta in renal injury and disease. Semin. Nephrol. 27, 309-320. doi: 10.1016/j.semnephrol.2007.02.009

Cassina, L., Chiaravalli, M., and Boletta, A. (2020). Increased mitochondrial fragmentation in polycystic kidney disease acts as a modifier of disease progression. FASEB J. 34, 6493-6507. doi: 10.1096/fj.201901739RR is not fully understood, it is likely that KCa3.1 regulates mitochondrial quality control through the modulation of membrane potential, cell volume, or calcium influx, which are crucial for mitochondrial function, mitochondrial dynamics, and mitophagy (Mohr et al., 2019; Romero-Garcia and PradoGarcia, 2019). The findings from the current study not only further confirm the role of mitochondrial dysfunction in diabetic kidney disease, but also offer the potential of targeting $\mathrm{KCa} 3.1$ to normalize mitochondrial quality control in the treatment of diabetic kidney disease.

\section{DATA AVAILABILITY STATEMENT}

The raw data supporting the conclusions of this article will be made available by the corresponding author, without undue reservation.

\section{ETHICS STATEMENT}

The animal study was reviewed and approved by the Animal Research Ethics Committee of Royal North Shore Hospital.

\section{AUTHOR CONTRIBUTIONS}

$\mathrm{CH}, \mathrm{CP}$, and $\mathrm{X}-\mathrm{MC}$ conceptualized and designed the experiments. $\mathrm{CH}, \mathrm{HY}, \mathrm{YS}, \mathrm{QC}$, YS, DC, and FB performed the experiments and analyzed the data. $\mathrm{CH}$ drafted the manuscript. All authors contributed to manuscript revision and read and approved the final version of the manuscript.

\section{FUNDING}

This research was supported by Juvenile Diabetes Research Foundation International.

\section{ACKNOWLEDGMENTS}

The KCa3.1-/- mice were kindly provided by Dr. James Melvin, National Institute of Dental and Craniofacial Research, Bethesda, MD, United States.

Chachi, L., Shikotra, A., Duffy, S. M., Tliba, O., Brightling, C., Bradding, P., et al. (2013). Functional KCa3.1 channels regulate steroid insensitivity in bronchial smooth muscle cells. J. Immunol. 191, 2624-2636. doi: 10.4049/jimmunol. 1300104

Chen, Y. J., Raman, G., Bodendiek, S., O’Donnell, M. E., and Wulff, H. (2011). The KCa3.1 blocker TRAM-34 reduces infarction and neurological deficit in a rat model of ischemia/reperfusion stroke. J. Cereb. Blood Flow Metab. 31, 2363-2374. doi: 10.1038/jcbfm.20 11.101

Choi, H. I., Park, J. S., Kim, D. H., Kim, C. S., Bae, E. H., Ma, S. K., et al. (2019). PGC-1alpha Suppresses the Activation of TGF-beta/Smad Signaling via Targeting TGFbetaRI Downregulation by let-7b/c Upregulation. Int. J. Mol. Sci. 20:202084. doi: 10.3390/ijms20205084 
Chou, C. C., Lunn, C. A., and Murgolo, N. J. (2008). KCa3.1: target and marker for cancer, autoimmune disorder and vascular inflammation? Expert Rev. Mol. Diagn. 8, 179-187. doi: 10.1586/14737159.8.2.179

Cruse, G., Duffy, S. M., Brightling, C. E., and Bradding, P. (2006). Functional $\mathrm{KCa} 3.1 \mathrm{~K}+$ channels are required for human lung mast cell migration. Thorax. 61, 880-885. doi: 10.1136/thx.2006.060319

De Marchi, U., Sassi, N., Fioretti, B., Catacuzzeno, L., Cereghetti, G. M., Szabo, I., et al. (2009). Intermediate conductance Ca2+-activated potassium channel (KCa3.1) in the inner mitochondrial membrane of human colon cancer cells. Cell Calcium. 45, 509-516. doi: 10.1016/j.ceca.2009.03.014

Del Dotto, V., Fogazza, M., Carelli, V., Rugolo, M., and Zanna, C. (2018). Eight human OPA1 isoforms, long and short: What are they for? Biochim. Biophys. Acta Bioenerg. 1859, 263-269. doi: 10.1016/j.bbabio.2018.01.005

Demeter-Haludka, V., Kovacs, M., Petrus, A., Patai, R., Muntean, D. M., Siklos, L., et al. (2018). Examination of the Role of Mitochondrial Morphology and Function in the Cardioprotective Effect of Sodium Nitrite Administered 24 h Before Ischemia/Reperfusion Injury. Front. Pharmacol. 9:286. doi: 10.3389/ fphar.2018.00286

Dhingra, A., Jayas, R., Afshar, P., Guberman, M., Maddaford, G., Gerstein, J., et al. (2017). Ellagic acid antagonizes Bnip3-mediated mitochondrial injury and necrotic cell death of cardiac myocytes. Free Radic. Biol. Med. 112, 411-422. doi: 10.1016/j.freeradbiomed.2017.08.010

Forbes, J. M., and Thorburn, D. R. (2018). Mitochondrial dysfunction in diabetic kidney disease. Nat. Rev. Nephrol. 14, 291-312. doi: 10.1038/nrneph.2018.9

Galluzzi, L., Kepp, O., and Kroemer, G. (2012). Mitochondria: master regulators of danger signalling. Nat. Rev. Mol. Cell Biol. 13, 780-788. doi: 10.1038/nrm3479

Gao, J., Jiang, J., Xie, F., and Chen, L. (2020). Bnip3 in mitophagy: novel insights and potential therapeutic target for diseases of secondary mitochondrial dysfunction. Clinica. Chimica. Acta. 2020:024. doi: 10.1016/j.cca.2020.02.024

Gong, L. L., Yang, S., Zhang, W., Han, F. F., Lv, Y. L., Wan, Z. R., et al. (2018). Akebia saponin D alleviates hepatic steatosis through BNip3 induced mitophagy. J. Pharmacol. Sci. 136, 189-195. doi: 10.1016/j.jphs.2017.11.007

Grunwell, J. R., Yeligar, S. M., Stephenson, S., Ping, X. D., Gauthier, T. W., Fitzpatrick, A. M., et al. (2018). TGF-betal Suppresses the Type I IFN Response and Induces Mitochondrial Dysfunction in Alveolar Macrophages. J. Immunol. 200, 2115-2128. doi: 10.4049/jimmunol.1701325

Hanna, R. A., Quinsay, M. N., Orogo, A. M., Giang, K., Rikka, S., and Gustafsson, A. B. (2012). Microtubule-associated protein 1 light chain 3 (LC3) interacts with Bnip3 protein to selectively remove endoplasmic reticulum and mitochondria via autophagy. J. Biol. Chem. 287, 19094-19104. doi: 10.1074/jbc.M111.322933

Hills, C. E., and Squires, P. E. (2011). The role of TGF-beta and epithelial-to mesenchymal transition in diabetic nephropathy. Cytokine Growth Factor Rev. 22, 131-139. doi: 10.1016/j.cytogfr.2011.06.002

Huang, C., Lin, M. Z., Cheng, D., Braet, F., Pollock, C. A., and Chen, X. M. (2014a). Thioredoxin-interacting protein mediates dysfunction of tubular autophagy in diabetic kidneys through inhibiting autophagic flux. Lab. Invest. 94, 309-320. doi: 10.1038/labinvest.2014.2

Huang, C., Lin, M. Z., Cheng, D., Braet, F., Pollock, C. A., and Chen, X. M. (2016a). KCa3.1 mediates dysfunction of tubular autophagy in diabetic kidneys via PI3k/Akt/mTOR signaling pathways. Sci. Rep. 6:23884. doi: 10.1038/srep23884

Huang, C., Shen, S., Ma, Q., Chen, J., Gill, A., Pollock, C. A., et al. (2013). Blockade of KCa3.1 ameliorates renal fibrosis through the TGF-beta1/Smad pathway in diabetic mice. Diabetes. 62, 2923-2934. doi: 10.2337/db13-0135

Huang, C., Shen, S., Ma, Q., Gill, A., Pollock, C. A., and Chen, X. M. (2014b). KCa3.1 mediates activation of fibroblasts in diabetic renal interstitial fibrosis. Nephrol. Dial Transplant. 29, 313-324. doi: 10.1093/ndt/gft431

Huang, C., Zhang, Y., Kelly, D. J., Tan, C. Y., Gill, A., Cheng, D., et al. (2016b). Thioredoxin interacting protein (TXNIP) regulates tubular autophagy and mitophagy in diabetic nephropathy through the mTOR signaling pathway. Sci. Rep. 6:29196. doi: 10.1038/srep29196

Ishihara, M., Urushido, M., Hamada, K., Matsumoto, T., Shimamura, Y., Ogata, K., et al. (2013). Sestrin-2 and BNIP3 regulate autophagy and mitophagy in renal tubular cells in acute kidney injury. Am. J. Physiol. Renal. Physiol. 305, F495-F509. doi: 10.1152/ajprenal.00642.2012

Kanzawa, T., Zhang, L., Xiao, L. I, Germano, M., Kondo, Y., and Kondo, S. (2005). Arsenic trioxide induces autophagic cell death in malignant glioma cells by upregulation of mitochondrial cell death protein BNIP3. Oncogene. 24, 980-991. doi: 10.1038/sj.onc. 1208095
Kovalenko, A. I, Glasauer, L., Schockel, D. R., Sauter, A., Ehrmann, F., Sohler, et al. (2016). Identification of KCa3.1 Channel as a Novel Regulator of Oxidative Phosphorylation in a Subset of Pancreatic Carcinoma Cell Lines. PLoS One. 11:e0160658. doi: 10.1371/journal.pone.0160658

Kubli, D. A., Ycaza, J. E., and Gustafsson, A. B. (2007). Bnip3 mediates mitochondrial dysfunction and cell death through Bax and Bak. Biochem. J. 405, 407-415. doi: 10.1042/BJ20070319

Lan, H. Y. (2012). Transforming growth factor-beta/Smad signalling in diabetic nephropathy. Clin. Exp. Pharmacol. Physiol. 39, 731-738. doi: 10.1111/j.14401681.2011.05663.x

Leanza, L., Zoratti, M., Gulbins, E., and Szabo, I. (2014). Mitochondrial ion channels as oncological targets. Oncogene 33, 5569-5581. doi: 10.1038/onc. 2013.578

Lee, S. Y., Kang, J. M., Kim, D. J., Park, S. H., Jeong, H. Y., Lee, Y. H., et al. (2017). PGC1alpha Activators Mitigate Diabetic Tubulopathy by Improving Mitochondrial Dynamics and Quality Control. J. Diabetes Res. 2017, 6483572. doi: 10.1155/2017/6483572

Li, J., Li, N., Yan, S., Lu, Y., Miao, X., Gu, Z., et al. (2019). Melatonin attenuates renal fibrosis in diabetic mice by activating the AMPK/PGC1alpha signaling pathway and rescuing mitochondrial function. Mol. Med. Rep. 19, 1318-1330. doi: 10.3892/mmr.2018.9708

Li, R., Xin, T., Li, D., Wang, C., Zhu, H., and Zhou, H. (2018). Therapeutic effect of Sirtuin 3 on ameliorating nonalcoholic fatty liver disease: The role of the ERK-CREB pathway and Bnip3-mediated mitophagy. Redox Biol. 18, 229-243. doi: 10.1016/j.redox.2018.07.011

Li, W., Du, M., Wang, Q., Ma, X., Wu, L., Guo, F., et al. (2017). FoxO1 Promotes Mitophagy in the Podocytes of Diabetic Male Mice via the PINK1/Parkin Pathway. Endocrinology. 158, 2155-2167. doi: 10.1210/en.2016-1970

Liu, X., Lu, J., Liu, S., Huang, D., Chen, M., Xiong, G., et al. (2020). HuangqiDanshen decoction alleviates diabetic nephropathy in $\mathrm{db} / \mathrm{db}$ mice by inhibiting PINK1/Parkin-mediated mitophagy. Am. J Transl. Res. 12, 989-998.

Liu, Z., Liu, H., Xiao, L., Liu, G., Sun, L., and He, L. (2019). STC-1 ameliorates renal injury in diabetic nephropathy by inhibiting the expression of BNIP3 through the AMPK/SIRT3 pathway. Lab. Invest. 99, 684-697. doi: 10.1038/s41374-0180176-7

Lomash, R. M., Petralia, R. S., Holtzclaw, L. A., Tsuda, M. C., Wang, Y. X., Badger, J. D. II, et al. (2019). Neurolastin, a dynamin family GTPase, translocates to mitochondria upon neuronal stress and alters mitochondrial morphology in vivo. J. Biol. Chem. 294, 11498-11512. doi: 10.1074/jbc.RA118.007245

Meng, X. M., Chung, A. C., and Lan, H. Y. (2013). Role of the TGF-beta/BMP7/Smad pathways in renal diseases. Clin. Sci. 124, 243-254. doi: 10.1042/ CS20120252

Mohr, C. J., Steudel, F. A., Gross, D., Ruth, P., Lo, W. Y., Hoppe, R., et al. (2019). Cancer-Associated Intermediate Conductance $\mathrm{Ca}(2+)$-Activated $\mathrm{K}(+)$ Channel KCa3.1. Cancers 11:11010109. doi: 10.3390/cancers11010109

Nguyen, L. T., Stangenberg, S., Chen, H., Al-Odat, I., Chan, Y. L., Gosnell, M. E., et al. (2015). L-Carnitine reverses maternal cigarette smoke exposure-induced renal oxidative stress and mitochondrial dysfunction in mouse offspring. Am. J. Physiol. Renal. Physiol. 308, F689-F696. doi: 10.1152/ajprenal.00417.2014

Patel, A. S., Song, J. W., Chu, S. G., Mizumura, K., Osorio, J. C., Shi, Y., et al. (2015). Epithelial cell mitochondrial dysfunction and PINK1 are induced by transforming growth factor-betal in pulmonary fibrosis. PLoS One 10:e0121246. doi: 10.1371/journal.pone.0121246

Pozdzik, A. A., Giordano, L., Li, G., Antoine, M. H., Quellard, N., Godet, J., et al. (2016). Blocking TGF-beta Signaling Pathway Preserves Mitochondrial Proteostasis and Reduces Early Activation of PDGFRbeta+ Pericytes in Aristolochic Acid Induced Acute Kidney Injury in Wistar Male Rats. PLoS One 11:e0157288. doi: 10.1371/journal.pone.0157288

Ranjit, S., Dobrinskikh, E., Montford, J., Dvornikov, A., Lehman, A., Orlicky, D. J., et al. (2016). Label-free fluorescence lifetime and second harmonic generation imaging microscopy improves quantification of experimental renal fibrosis. Kidney Int. 90, 1123-1128. doi: 10.1016/j.kint.2016.06.030

Romero-Garcia, S., and Prado-Garcia, H. (2019). Mitochondrial calcium: Transport and modulation of cellular processes in homeostasis and cancer (Review). Int. J. Oncol. 54, 1155-1167. doi: 10.3892/ijo.2019.4696

Saxena, S., Mathur, A., and Kakkar, P. (2019). Critical role of mitochondrial dysfunction and impaired mitophagy in diabetic nephropathy. J. Cell Physiol. 234, 19223-19236. doi: 10.1002/jcp.28712 
Sharma, K., McCue, P., and Dunn, S. R. (2003). Diabetic kidney disease in the $\mathrm{db} / \mathrm{db}$ mouse. Am. J. Physiol. Renal. Physiol. 284, F1138-F1144. doi: 10.1152/ajprenal. 00315.2002

Suarez-Rivero, J. M., Villanueva-Paz, M., de la Cruz-Ojeda, P., de la Mata, M., Cotan, D., Oropesa-Avila, M., et al. (2016). Mitochondrial Dynamics in Mitochondrial Diseases. Diseases 5:5010001. doi: 10.3390/diseases5010001

Sun, L., Yuan, Q., Xu, T., Yao, L., Feng, J., Ma, J., et al. (2017). Pioglitazone Improves Mitochondrial Function in the Remnant Kidney and Protects against Renal Fibrosis in 5/6 Nephrectomized Rats. Front. Pharmacol. 8:545. doi: 10.3389/ fphar.2017.00545

Sun, Q., Fang, L., Tang, X., Lu, S., Tamm, M., Stolz, D., et al. (2019). TGFbeta Upregulated Mitochondria Mass through the SMAD2/3->C/EBPbeta$>$ PRMT1 Signal Pathway in Primary Human Lung Fibroblasts. J. Immunol. 202, 37-47. doi: 10.4049/jimmunol.1800782

Suomalainen, A., and Battersby, B. J. (2018). Mitochondrial diseases: the contribution of organelle stress responses to pathology. Nat. Rev. Mol. Cell Biol. 19, 77-92. doi: 10.1038/nrm.2017.66

Tang, C., Han, H., Liu, Z., Liu, Y., Yin, L., Cai, J., et al. (2019). Activation of BNIP3mediated mitophagy protects against renal ischemia-reperfusion injury. Cell Death Dis. 10:677. doi: 10.1038/s41419-019-1899-0

Wang, J., Zhu, P., Li, R., Ren, J., and Zhou, H. (2020). Fundcl-dependent mitophagy is obligatory to ischemic preconditioning-conferred renoprotection in ischemic AKI via suppression of Drp1-mediated mitochondrial fission. Redox Biol. 30:101415. doi: 10.1016/j.redox.2019.101415

Wang, Y., Lu, M., Xiong, L., Fan, J., Zhou, Y., Li, H., et al. (2020). Drp1-mediated mitochondrial fission promotes renal fibroblast activation and fibrogenesis. Cell Death Dis. 11:29. doi: 10.1038/s41419-019-2218-5

Wei, X., Wei, X., Lu, Z., Li, L., Hu, Y., Sun, F., et al. (2020). Activation of TRPV1 channel antagonizes diabetic nephropathy through inhibiting endoplasmic reticulum-mitochondria contact in podocytes. Metabolism. 105:154182. doi: 10.1016/j.metabol.2020.154182

Wulff, H., and Castle, N. A. (2010). Therapeutic potential of KCa3.1 blockers: recent advances and promising trends. Expert Rev. Clin. Pharmacol. 3, 385-396. doi: $10.1586 /$ ecp. 10.11

Wulff, H., Kolski-Andreaco, A., Sankaranarayanan, A., Sabatier, J. M., and Shakkottai, V. (2007). Modulators of small- and intermediate-conductance calcium-activated potassium channels and their therapeutic indications. Curr. Med. Chem. 14, 1437-1457.

Xiao, L., Xu, X., Zhang, F., Wang, M., Xu, Y., Tang, D., et al. (2017). The mitochondria-targeted antioxidant MitoQ ameliorated tubular injury mediated by mitophagy in diabetic kidney disease via Nrf2/PINK1. Redox Biol. 11, 297-311. doi: 10.1016/j.redox.2016.12.022

Yu, J., Mao, S., Zhang, Y., Gong, W., Jia, Z., Huang, S., et al. (2016). MnTBAP Therapy Attenuates Renal Fibrosis in Mice with 5/6 Nephrectomy. Oxid Med. Cell Longev. 2016:7496930. doi: 10.1155/2016/7496930

Zhan, M., Brooks, C., Liu, F., Sun, L., and Dong, Z. (2013). Mitochondrial dynamics: regulatory mechanisms and emerging role in renal pathophysiology. Kidney Int. 83, 568-581. doi: 10.1038/ki.2012.441

Zhang, Q., He, L., Dong, Y., Fei, Y., Wen, J., Li, X., et al. (2020). Sitagliptin ameliorates renal tubular injury in diabetic kidney disease via STAT3-dependent mitochondrial homeostasis through SDF-1alpha/CXCR4 pathway. FASEB J. 34, 7500-7519. doi: 10.1096/fj.201903038R

Zhang, Y., Ma, Y., Liang, N., Liang, Y., Lu, C., and Xiao, F. (2019). Blockage of ROS-ERK-DLP1 signaling and mitochondrial fission alleviates $\mathrm{Cr}(\mathrm{VI})$ induced mitochondrial dysfunction in L02 hepatocytes. Ecotoxicol. Environ. Saf. 186:109749. doi: 10.1016/j.ecoenv.2019.109749

Zhao, C. Y., Chen, Z. Y., Xu, X. G., An, X. F., Duan, S. Y., Huang, Z. M., et al. (2017). Pink1/Parkin-mediated mitophagy play a protective role in cisplatin induced renal tubular epithelial cells injury. Exp. Cell Res. 350, 390-397. doi: 10.1016/j.yexcr.2016.12.015

Conflict of Interest: The authors declare that the research was conducted in the absence of any commercial or financial relationships that could be construed as a potential conflict of interest.

Copyright $\odot 2021$ Huang, Yi, Shi, Cao, Shi, Cheng, Braet, Chen and Pollock. This is an open-access article distributed under the terms of the Creative Commons Attribution License (CC BY). The use, distribution or reproduction in other forums is permitted, provided the original author(s) and the copyright owner(s) are credited and that the original publication in this journal is cited, in accordance with accepted academic practice. No use, distribution or reproduction is permitted which does not comply with these terms. 